\title{
The Planck List of High-z source candidates : A laboratory for high-z star-forming galaxies
}

\author{
L. Montier ${ }^{1,2}$ \\ on behalf of the Planck collaboration
}

${ }^{1}$ CNRS, IRAP, 9 Av. colonel Roche, BP 44346, F-31028 Toulouse cedex 4, France

${ }^{2}$ Université de Toulouse, UPS-OMP, IRAP, F-31028 Toulouse cedex 4, France

\begin{abstract}
The Planck satellite (Planck 2015 results. I) has provided the first FIR/submm all-sky survey with a sensitivity allowing us to identify the rarest, most luminous hig-z dusty star-forming sources on the sky. The Planck list of high-z source candidates (PHZ, PIP XXXIX subm) has been built and charcaterized over $25 \%$ of the sky by selecting the 2151 brightest red submm sources at a 5 ' resolution (Montier et al. 2010). Follow-up observations with Herschel/SPIRE over 228 Planck candidates have already shown that $93 \%$ of these candidates are actually overdensities of red sources (PIP XXVII 2015), while 12 Planck high-z candidates are identified as strongly lensed star-forming galaxies at redshift between 2.2 and 3.6 (Canameras et al. 2015). The first confirmed Planck proto-cluster candidate has been revealed to be a double structure at $\mathrm{z}=1.7$ and $\mathrm{z} z=2.03$ (Flores-Cacho et al. 2015). The PHZ opens a new window on these extreme star-forming systems at high-z, providing a powerful laboratory to study the mechanisms of galaxy evolution and enrichment in the frame of the large scale structure growth.
\end{abstract}

Keywords. submillimeter, galaxies: high-redshift, stars: formation, galaxies: clusters: general, cosmology: large-scale structure of universe

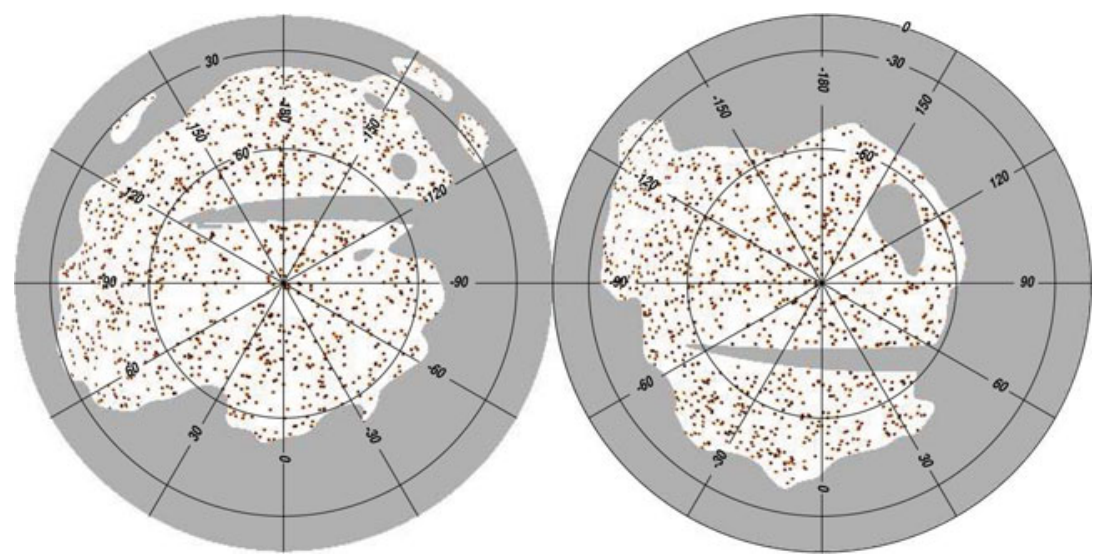

Figure 1. All-sky distribution of the PHZ sources

\section{References}

Canameras, I., Nesvadba, N., Guery, D. et al. 2015 A $\& A$, 581,A105

Flores-cacho, I., Pierini, D., Soucail, G. et al. A $\mathscr{E} A$ in press, arXiv:1510.01585

Montier, L. A., Pelkonen, V., Juvela, M., Ristorcelli, I., \& Marshall, D. J., 2010, A 8 A, 522, A83

Planck Collaboration, Planck 2015 results. I, 2015, subm to $A \& A$, arXiv:1502.01582

Planck Collaboration, Planck int. results. XXVII, 2015, A\& $A$, 582, A31

Planck Collaboration, Planck int. results. XXXIX, 2015, subm to $A \mathscr{E} A$, arXiv:1508.04171 